\title{
Z Boson Pair Exclusive Production from Two Photon Exchange with Anomalous Gauge Boson Couplings in $p$ p Collisions at $13 \mathrm{TeV}$
}

\author{
Jose SA BORGES* \\ Universidade do Estado do Rio de Janeiro \\ E-mail: jose.saborges@gmail.com \\ D. E. Martins \\ Universidade do Estado do Rio de Janeiro \\ E-mail: dan.ernaniegmail.com
}

\section{A. Vilela Pereira \\ Universidade do Estado do Rio de Janeiro \\ E-mail: antonio.vilela.pereira@cern.ch}

\begin{abstract}
A simulation for the existence of particles and couplings, that are not predicted in the Standard Model (SM), can be performed by evaluating the contributions of anomalous photon-gauge boson couplings to $\mathrm{W}$ and $\mathrm{Z}$ pair production in proton-proton collisions at the LHC. In this work, we present our preliminary results for $Z$ pair exclusive production from two-photon exchange with anomalous gauge boson quartic couplings, by considering a $1 \mathrm{TeV}$ scale for new physical effects. We consider some values for the integrated luminosity up to $100 \mathrm{fb}^{-1}$ at center-of-mass energy of $13 \mathrm{TeV}$. We calculate the total cross section for the exclusive process and we present the resulting four lepton invariant mass distribution. Finally we present an outlook for the present analysis.
\end{abstract}

XXVII International Symposium on Lepton Photon Interactions at High Energies 17-22 August 2015

Ljubljana, Slovenia

\footnotetext{
* Speaker.
} 


\section{Introduction}

At the Large Hadron Collider (LHC), $p p$ collisions produce a significant number of highenergy quasi-real photons. Hence, LHC can be somehow considered as a high-energy photonphoton or photon-proton collider, allowing for a standard luminosity calibration. In this conjecture, the small effective luminosity is compensated by the knowledge of the initial condition associated with a clear experimental signature of the produced particles. In particular the presence of very forward scattered protons, to be explored by the new dedicated detectors such as the CMS-TOTEM Precision Proton Spectrometer (CT-PPS) at CMS [1], helps the identification of the relevant signal. Another important aspect is related to the simulation of new physical effects, by introducing anomalous couplings of photons with gauge bosons [2].

Anomalous couplings can be introduced by building an effective Lagrangian which models the low energy behavior of possible extensions of the SM. Here we introduce the so-called genuine quartic anomalous couplings by adding new interaction terms via effective dimension- 6 operators conserving local $\mathrm{U}(1)_{e m}$ and custodial $\mathrm{SU}(2)_{c}$ symmetries.

We start by discussing the Equivalent Photon Approximation (EPA) which lies on the hypothesis of factorization of the two-photon cross section from the two photon fluxes [3]. Next we present the effective Lagrangian and the procedure to calculate the matrix elements, as well as to simulate the production cross section using the FPMC [4] events generator. Our results are shown for $Z$ pair production with a brief discussion on background contributions.

\section{Equivalent Photon Approximation}

The exclusive production of gauge boson pairs is a photon-induced process that happens when each incoming proton emits one low virtuality photon $\left(Q^{2}<2 \mathrm{GeV}^{2}\right)$ that interacts producing an object $X$ characterized by the available two-photon energy $W_{\gamma \gamma}$. Since the average photon virtualities are very low, one can safely treat all such processes as quasi-real photon interactions [5].

The factorization of the amplitude of a given process as the product of a photon exchange part by the photon interaction part allows us to use the Equivalent Photon Approximation (EPA) to describe $Z$ pair production from two-photon exchange. For $Z Z$ quartic interactions with two photons, that are absent in the SM Lagrangian, we introduce an effective Lagrangian with two anomalous couplings $a_{0}^{Z}$ and $a_{C}^{Z}$ and an energy scale of new physics, $\Lambda \sim 1 \mathrm{TeV}$ [6].

$$
\mathscr{L}_{6}^{0}=\frac{e^{2}}{16 \cos ^{2} \theta_{W}} \frac{a_{0}^{Z}}{\Lambda^{2}} F_{\mu \nu} F^{\mu v} Z^{\alpha} Z_{\alpha}+\frac{e^{2}}{16 \cos ^{2} \theta_{W}} \frac{a_{C}^{Z}}{\Lambda^{2}} F_{\mu \alpha} F^{\mu \beta} Z^{\alpha} Z_{\beta}
$$

Let us recall that an exclusive process is characterized by an empty region of pseudo-rapidity that occurs when the forward protons are detected apart from the decay particles of the massive produced object [7]. In particular, for the two-photon exchange in $p p$ collisions considered here is a pair of $Z$ s decaying into leptons. The expected experimental topology corresponding to the signal is characterized by three aspects. First the production of very forward protons that remain intact, second the presence of very few particles in the central region and finally the production of leptons with high transverse momentum. 


\section{Procedure and Results}

The present analysis of $Z$ pair production from two photon interaction requires photon-gauge boson anomalous coupling, because the quartic coupling $\gamma \gamma Z Z$ is not present in the SM Lagrangian. For the signal we calculate two pair of $e^{+} e^{-}$or $\mu^{+} \mu^{-}$or one pair of $e^{+} e^{-}$and one pair of $\mu^{+} \mu^{-}$ produced by two photons coupled with two $Z$ s as given by $\mathscr{L}^{0, C}$.

We employ the Forward Physics Monte Carlo (FPMC) package for event generation, where we apply minimal cuts for each lepton transverse momentum $p_{T}>10 \mathrm{GeV}$ and $|\eta|<2.5$ for the lepton pseudo-rapidity. We perform the selection of the final state by using the HepMC event record format [8]. Then, we write a macro in the ROOT framework [9] to choose the highest transverse momentum leptons to produce the final observables and distributions.

We consider the proton momentum lost in the interval $0.015<\xi_{i}<0.17, i=1,2$ for the signal, where we select one lepton pair with the highest transverse momentum such that their momenta combination corresponds to an on-shell $Z$; these selected pair do not participate in the selection of the other pair with combined momenta corresponding to $M_{Z} \pm 10 \mathrm{GeV}$. We define the central mass by $M_{X}^{2}=\xi_{1} \xi_{2} s$, for $\sqrt{s}=13 \mathrm{TeV}$ and we present the contribution of anomalous photon-gauge boson couplings for $Z$ pair production.

The results of four lepton mass distribution corresponding to a muon-anti-muon pair and a electron-positron pair, when all mentioned cuts are implemented is shown in the left panel of figure 1 ; the $\gamma \gamma$ central mass is shown in the right side of same figure.
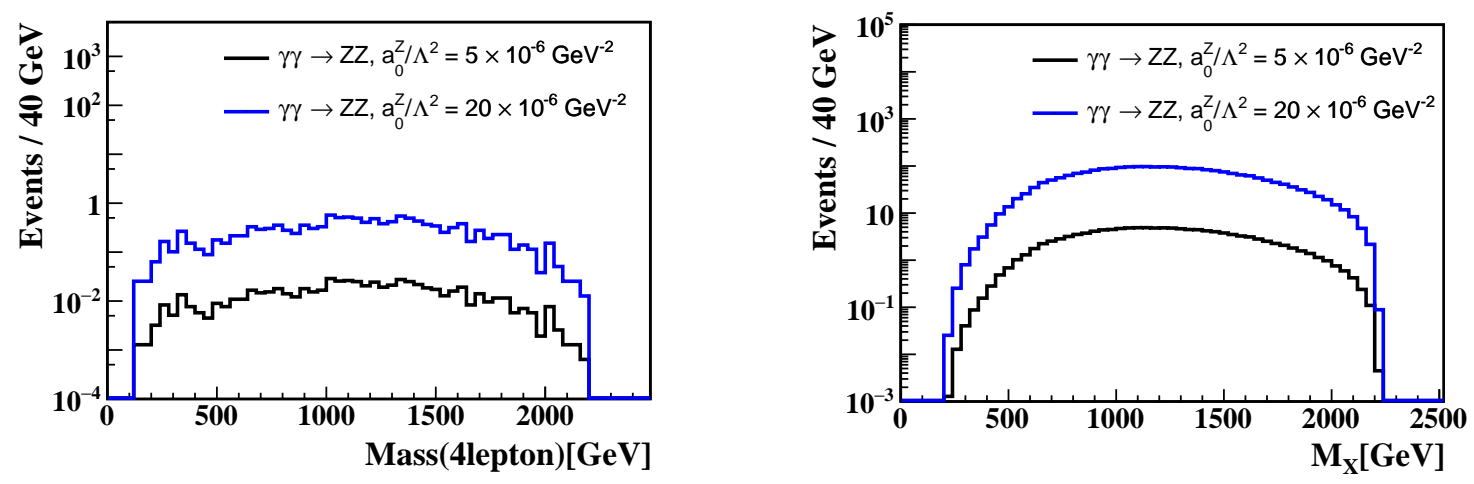

Figure 1: Left: The four lepton mass distributions. Right: Central mass distributions. These results are in pp collision at $13 \mathrm{TeV}$ for $100 \mathrm{fb}^{-1}$, for two values of photon-gauge boson anomalous coupling.

In addition we calculate the ratio $\mathrm{R}_{\text {sign }}$ of the expected number of four leptons satisfying the cuts $N_{\text {signal }}$ and the total number of double pair produced. We summarize this analysis with a table that shows the expected number $N_{\text {signal }}$ of two pairs of leptons in the four lepton mass distribution, and the value of $R$.

\begin{tabular}{|l|l|l|l|}
\hline & $\mathrm{L}=30$ & $\mathrm{~L}=50$ & $\mathrm{~L}=100$ \\
\hline$N_{\text {signal }}$ & 0.22 & 0.33 & 0.67 \\
\hline$R_{\text {sign }}$ & 0.98 & 1.03 & 1.4 \\
\hline
\end{tabular}

\begin{tabular}{|l|l|l|l|}
\hline & $\mathrm{L}=30$ & $\mathrm{~L}=50$ & $\mathrm{~L}=100$ \\
\hline$N_{\text {signal }}$ & 3.9 & 6.6 & 13.1 \\
\hline$R_{\text {sign }}$ & 17 & 20 & 27 \\
\hline
\end{tabular}

Table 1: Number signal events and the ratio $R$ for three values of the integrated luminosity given in $f b^{-1}$. Left for $a_{0}^{Z} / \Lambda=5 \times 10^{-6} \mathrm{GeV}^{-2}$. Right for $a_{0}^{Z} / \Lambda=20 \times 10^{-6} \mathrm{GeV}^{-2}$. 

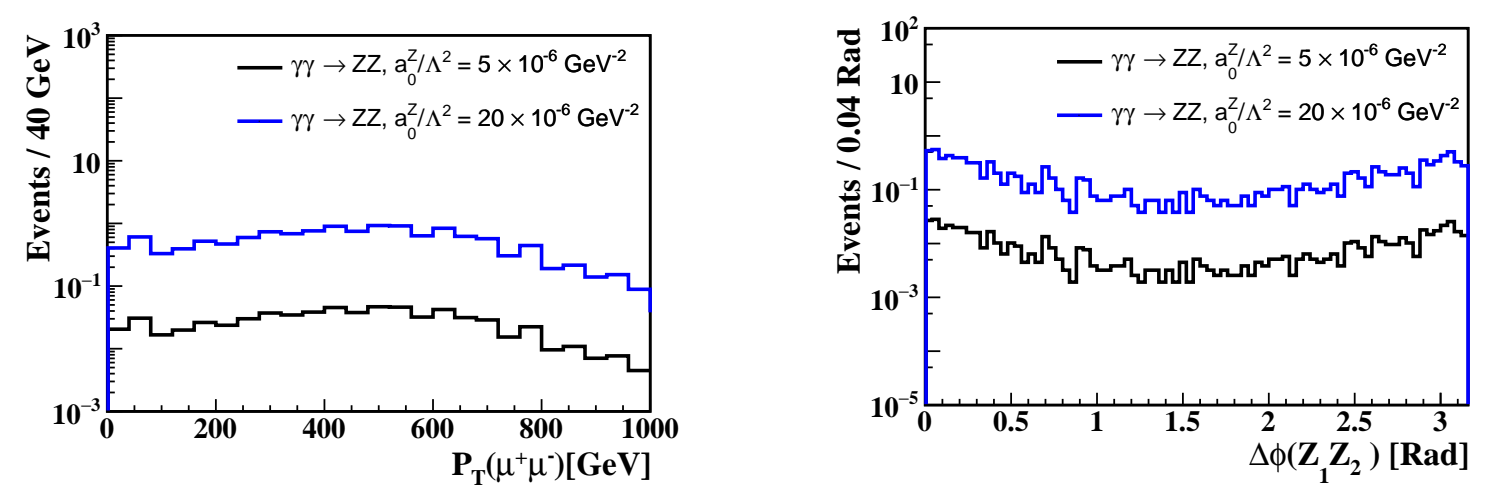

Figure 2: Left: Transverse momentum distributions for a muon-anti-muon pair in pp collision at $13 \mathrm{TeV}$ for $100 \mathrm{fb}^{-1}$. Right: Angular azimutal deviation of the two lepton pairs.

\section{Comments and Perspectives}

We analyze the exclusive production of a pair of $Z$ s from two-photon exchange in $p p$ collisions at the LHC for $\sqrt{s}=13 \mathrm{TeV}$ for integrated luminosity up to $100 \mathrm{fb}^{-1}$. We introduce two anomalous coupling parameter in the Lagrangian $\mathscr{L}_{6}^{0, C}$ to show that the presence of anomalous couplings can be identified from the four lepton mass distributions.

These are preliminary results, to be complemented by a deep evaluation of backgrounds processes. On the other hand, we intend to use a specific model, with additional gauge bosons to the SM ones, to compare its result with those following from effective anomalous couplings.

\section{References}

[1] CMS and TOTEM Collaborations, Tech. Rep. CERN-LHCC-2014-021, TOTEM-TDR-003, CMS-TDR-13, CERN, Geneva (2014).

[2] O. J. P. Eboli, M. C. Gonzalez-Garcia, S. M. Lietti and S. F. Novaes, Phys. Rev. D 63, 075008 (2001).

[3] V. M. Budnev et al., Phys. Rep. 15C, 181 (1975).

[4] M. Boonekamp, A. Dechambre, arXiv:0903.3861 [hep-ph]; http://cern.ch/fpmc.

[5] J. de Favereau et al., CP3-09-37 (2009).

[6] G. Belanger and F. Boudjema, Phys. Lett. B 288, 201 (1992); Phys. Lett. B 288, 210 (1992).

[7] C. Royon, E. Chapon, and O. Kepka, PoS EPS-HEP2009 380 (2009).

[8] M. Dobbs, and J. B Hensen, Comput. Phys. Commun. 134, pp. 41-46, (2001).

[9] R. Brun and F. Rademakers, Nucl. Inst. \& Meth. in Phys. Res. A AIHENP'96 Workshop, Lausane, 81-86, (1996). 